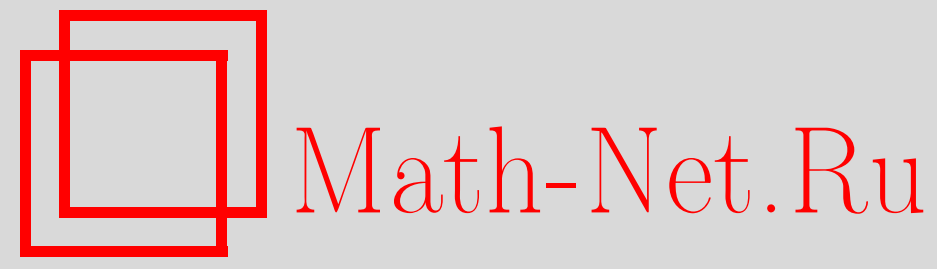

Г. Г. Магарил-Ильяев, К. Ю. Осипенко, Оптимальное восстановление функций и их производных по приближенной информации о спектре и неравенства для производных, Функи. анализ и его прил., 2003, том 37, выпуск 3, 51-64

DOI: https://doi.org/10.4213/faa157

Использование Общероссийского математического портала MathNet.Ru подразумевает, что вы прочитали и согласны с пользовательским соглашением

http://www . mathnet.ru/rus/agreement

Параметры загрузки:

IP : 3.85 .73 .92

26 апреля 2023 г., $17: 12: 43$

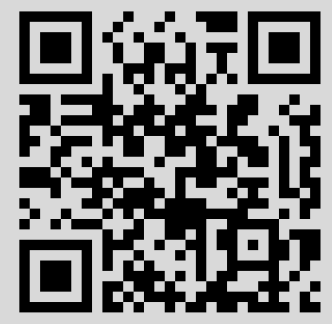


Функциональный анализ и его приложения

2003, т. 37, вып. 3, с. 51-64

УДК 517.51

\title{
Оптимальное восстановление функций и их производных по приближенной информации о спектре и неравенства для производных*
}

\author{
(C) 2003. Г. Г. МАГАРИЛ-ИЛЬЯЕВ, К. Ю. ОСИПЕНКО
}

\section{§1. Постановка задачи}

Начнем с общей постановки задачи об оптимальном восстановлении. Пусть $C$ - некоторое множество (класс) в векторном пространстве $X$. Про каждый элемент $x \in C$ мы располагаем информацией $I(x)$, где $I$ - отображение (называемое информационным) из $C$ в другое векторное пространство $Y$. Информация об элементах из $C$ может быть задана неточно, и потому $I$, вообще говоря, многозначное отображение. Пусть, далее, $Z-$ нормированное пространство и $\Lambda: X \rightarrow Z-$ линейный оператор. Задача заключается в том, чтобы восстановить (по возможности наилучшим образом) оператор $\Lambda$ на классе $C$ в метрике пространства $Z$ по имеющейся информации $I$. В это вкладывается следующий смысл. Любое отображение $\varphi: Y \rightarrow Z$ будем называть методом восстановления (оператора $\Lambda$ на классе $C$ по информации $I$ ). Погрешностью этого метода называется величина

Величина

$$
e(\Lambda, C, I, \varphi)=\sup _{x \in C, y \in I(x)}\|\Lambda x-\varphi(y)\|_{Z} .
$$

$$
E(\Lambda, C, I)=\inf _{\varphi: Y \rightarrow Z} e(\Lambda, C, I, \varphi),
$$

где нижняя грань берется по всем отображениям $\varphi: Y \rightarrow Z$, носит название погрешности оптимального восстановления, а метод, на котором достигается эта нижняя грань, называется оптимальным методом восстановления.

Впервые задача оптимального восстановления была поставлена С. А. Смоляком [1] для случая, когда $Z=\mathbb{R}, I-$ линейный оператор и $\operatorname{dim} Y<\infty$. Впоследствии вся эта проблематика интенсивно развивалась в разных направлениях (см. [2-5]). Подход к задачам восстановления, основанный на общих принципах теории экстремума, развивался в работах [6-8]. Этот подход мы используем и в данной статье.

Перейдем к постановке задач восстановления, рассматриваемых в работе. Пусть $S$ - пространство Шварца быстро убывающих бесконечно дифференцируемых функций на $\mathbb{R}, S^{\prime}-$ соответствующее пространство обобщенных функций, $F: S^{\prime} \rightarrow S^{\prime}-$ преобразование Фурье, $1 \leqslant p \leqslant \infty$ и $n \in \mathbb{N}$. В качестве пространства $X$ в задаче (1) будем рассматривать пространство

$$
X_{p}^{n}=\left\{x \in S^{\prime} \mid F x(\cdot) \in L_{p}(\mathbb{R}), x^{(n)}(\cdot) \in L_{2}(\mathbb{R})\right\},
$$

* Работа выполнена при финансовой поддержке Российского фонда фундаментальных исследований (гранты № 00-15-96109 и № 02-01-00386), программы «Университеты России» (УР.04.03.013), а также при поддержке U.S.CRDF-R.F.Ministry of Education Award VZ-0100-0. 
а в качестве класса $C-$ множество

$$
C_{p}^{n}=\left\{x(\cdot) \in X_{p}^{n}:\left\|x^{(n)}(\cdot)\right\|_{L_{2}(\mathbb{R})} \leqslant 1\right\} .
$$

Опишем информационные отображения, которые здесь изучаются. Пусть $1 \leqslant$ $p<\infty, 0<\sigma \leqslant \infty, \Delta_{\sigma}=(-\sigma, \sigma)$ и $\delta>0$. Будем считать, что информация об элементе $x(\cdot) \in C_{p}^{n}$ состоит в том, что нам известна функция $y(\cdot) \in L_{p}\left(\Delta_{\sigma}\right)$, такая, что $\|F x(\cdot)-y(\cdot)\|_{L_{p}\left(\Delta_{\sigma}\right)} \leqslant \delta$. Таким образом, информационное отображение $I=I_{p}^{\delta, \sigma}: C_{p}^{n} \rightarrow L_{p}\left(\Delta_{\sigma}\right)$ таково: $I_{p}^{\delta, \sigma} x(\cdot)=\left.F x(\cdot)\right|_{\Delta_{\sigma}}+\delta B L_{p}\left(\Delta_{\sigma}\right)$, где $B L_{p}\left(\Delta_{\sigma}\right)$ - единичный шар в $L_{p}\left(\Delta_{\sigma}\right)$.

Если $p=\infty$, то мы рассматриваем несколько более общую ситуацию. Пусть $\delta(\cdot)$ - неотрицательная функция из $L_{\infty}\left(\Delta_{\sigma}\right)$. Будем считать, что информация об элементе $x(\cdot)$ заключается в том, что известна функция $y(\cdot) \in L_{\infty}\left(\Delta_{\sigma}\right)$, такая, что $|F x(t)-y(t)| \leqslant \delta(t)$ для п. в. $t \in \Delta_{\sigma}$. Если положить

$$
B(\delta(\cdot))=\left\{y(\cdot) \in L_{\infty}\left(\Delta_{\sigma}\right):|y(t)| \leqslant \delta(t) \text { п. в. }\right\},
$$

то информационное отображение $I=I_{\infty}^{\delta(\cdot), \sigma}: C_{\infty}^{n} \rightarrow L_{\infty}\left(\Delta_{\sigma}\right)$ в этом случае имеет вид $I_{\infty}^{\delta(\cdot), \sigma} x(\cdot)=\left.F x(\cdot)\right|_{\Delta_{\sigma}}+B(\delta(\cdot))$.

Ставится задача об оптимальном восстановлении линейного оператора $\Lambda$, $\Lambda x(\cdot)=x^{(k)}(\cdot), 0 \leqslant k \leqslant n-1$, на классе $C_{p}^{n}$ в метрике пространства $L_{2}(\mathbb{R})$ по описанным выше информационным отображениям (далее будет показано, что $\Lambda: X_{p}^{n} \rightarrow L_{2}(\mathbb{R})$ при $\left.2 \leqslant p \leqslant \infty\right)$.

Таким образом, в нашем случае задача (1) при $p<\infty$ приобретает вид

$$
E\left(x^{(k)}(\cdot), C_{p}^{n}, I_{p}^{\delta, \sigma}\right)=\inf _{\varphi} \sup _{\substack{x(\cdot) \in C_{p}^{n}, y(\cdot) \in L_{p}\left(\Delta_{\sigma}\right) \\\|F x(\cdot)-y(\cdot)\|_{L_{p}\left(\Delta_{\sigma}\right)} \leqslant \delta}}\left\|x^{(k)}(\cdot)-\varphi(y)(\cdot)\right\|_{L_{2}(\mathbb{R})},
$$

где нижняя грань берется по всем $\varphi: L_{p}\left(\Delta_{\sigma}\right) \rightarrow L_{2}(\mathbb{R})$. Если $p=\infty$, то ее вид таков:

$$
E\left(x^{(k)}(\cdot), C_{\infty}^{n}, I_{\infty}^{\delta(\cdot), \sigma}\right)=\inf _{\varphi} \sup _{\substack{x(\cdot) \in C_{\infty}^{n}, y(\cdot) \in L_{\infty}\left(\Delta_{\sigma}\right) \\|F x(t)-y(t)| \leqslant \delta(t) \text { ח. в. }}}\left\|x^{(k)}(\cdot)-\varphi(y)(\cdot)\right\|_{L_{2}(\mathbb{R})},
$$

где нижняя грань берется по всем $\varphi: L_{\infty}\left(\Delta_{\sigma}\right) \rightarrow L_{2}(\mathbb{R})$.

В задаче (2) при $p=2$ и в задаче (3) мы находим точные значения для погрешности оптимального восстановления и явные выражения для оптимальных методов восстановления. В обоих случаях наблюдается следующее явление: для фиксированной погрешности в задаче $(3)$ существует такое конечное $\hat{\sigma}>0$, что знание преобразования Фурье на интервале, большем, чем $(-\hat{\sigma}, \hat{\sigma})$, не приводит к уменьшению погрешности оптимального восстановления. Этот вывод представляется нам важным для практических приложений полученных здесь результатов.

В задаче (2) при $p=2$ и $\sigma=\infty$ информация равносильна (в силу теоремы Планшереля) тому, что известна сама функция с точностью до $\delta$ в метрике пространства $L_{2}(\mathbb{R})$. В такой постановке задача решена в [9]. Периодические аналоги задачи (2) при $p=2$ и задачи (3), а также их обобщения на более широкие классы функций изучались в работе [10].

При $2<p<\infty$ получена оценка снизу величины (2). При этом доказано точное неравенство для $k$-й производной функции в $L_{2}$-норме, дающее ее оценку через $L_{2}$-норму $n$-й производной и $L_{p}$-норму ее преобразования Фурье. 


\section{§2. Формулировки основных результатов}

Teopema 1. Пусmb $n \in \mathbb{N}, 0 \leqslant k \leqslant n-1,0<\sigma \leqslant \infty, \Delta_{\sigma}=(-\sigma, \sigma)$, $\delta(\cdot) \in L_{\infty}\left(\Delta_{\sigma}\right), \delta(\cdot) \geqslant 0 u$

$$
\sigma_{0}=\sup \left\{a: 0<a<\sigma, \frac{1}{2 \pi} \int_{-a}^{a} t^{2 n} \delta^{2}(t) d t \leqslant 1\right\} .
$$

Тогда если $\sigma_{0}<\infty$, то

$$
E\left(x^{(k)}(\cdot), C_{\infty}^{n}, I_{\infty}^{\delta(\cdot), \sigma}\right)=\sqrt{\sigma_{0}^{-2(n-k)}+\frac{1}{2 \pi} \int_{-\sigma_{0}}^{\sigma_{0}}\left(t^{2 k}-\sigma_{0}^{-2(n-k)} t^{2 n}\right) \delta^{2}(t) d t}
$$

u

$$
\widehat{\varphi}(y)(t)=\frac{1}{2 \pi} \int_{-\sigma_{0}}^{\sigma_{0}}(i \tau)^{k}\left(1-\left(\frac{\tau}{\sigma_{0}}\right)^{2(n-k)}\right) y(\tau) e^{i \tau t} d \tau
$$

- оптимальный метод.

Если $\sigma_{0}=\infty$, то

$$
E\left(x^{(k)}(\cdot), C_{\infty}^{n}, I_{\infty}^{\delta(\cdot), \sigma}\right)=\sqrt{\frac{1}{2 \pi} \int_{-\infty}^{\infty} t^{2 k} \delta^{2}(t) d t}
$$

u

$$
\widehat{\varphi}(y)(t)=\frac{1}{2 \pi} \int_{-\infty}^{\infty}(i \tau)^{k} y(\tau) e^{i \tau t} d \tau
$$

- оптимальный метод.

СлЕДСТвиЕ 1. Пусть $\delta(t) \equiv \delta>0 u$

$$
\hat{\sigma}=(\pi(2 n+1))^{\frac{1}{2 n+1}} \delta^{-\frac{2}{2 n+1}} .
$$

Тогда

$$
E\left(x^{(k)}(\cdot), C_{\infty}^{n}, I_{\infty}^{\delta, \sigma}\right)= \begin{cases}\sqrt{\sigma^{-2(n-k)}+\frac{2 \delta^{2}(n-k)}{\pi(2 k+1)(2 n+1)} \sigma^{2 k+1}}, & \sigma<\hat{\sigma}, \\ \sqrt{\frac{2 n+1}{2 k+1}\left(\frac{1}{\pi(2 n+1)}\right)^{\frac{n-k}{2 n+1}} \delta^{\frac{2(n-k)}{2 n+1}},} & \sigma \geqslant \hat{\sigma},\end{cases}
$$

и метод (5) при $\sigma_{0}=\min (\sigma, \hat{\sigma})$ является оптимальным.

Из этого следствия вытекает, что при фиксированном $\delta$, начиная с $\hat{\sigma}$, дальнейшее увеличение интервала, на котором известно преобразование Фурье функции из $C_{\infty}^{n}$, заданное с погрешностью $\delta$ в равномерной метрике, не ведет к уменьшению погрешности восстановления. Другими словами, если нарушается соотношение

$$
\delta^{2} \sigma^{2 n+1} \leqslant \pi(2 n+1),
$$

связывающее исходные данные с величиной интервала, на котором эти данные измеряются, то получаемая информация оказывается уже избыточной.

Teopema 2. Пусть $n \in \mathbb{N}, 0<k \leqslant n-1,0<\sigma \leqslant \infty, \delta>0 u$

$$
\hat{\sigma}=\left(\frac{n}{k}\right)^{\frac{1}{2(n-k)}}\left(\frac{2 \pi}{\delta^{2}}\right)^{\frac{1}{2 n}} \text {. }
$$


Тогда

$$
E\left(x^{(k)}(\cdot), C_{2}^{n}, I_{2}^{\delta, \sigma}\right)= \begin{cases}\sigma^{k} \sqrt{\frac{n-k}{2 \pi n}\left(\frac{k}{n}\right)^{\frac{k}{n-k}} \delta^{2}+\frac{1}{\sigma^{2 n}}}, & \sigma<\hat{\sigma}, \\ \left(\frac{\delta^{2}}{2 \pi}\right)^{\frac{n-k}{2 n}}, & \sigma \geqslant \hat{\sigma},\end{cases}
$$

$u$

$$
\widehat{\varphi}(y)(t)=\frac{1}{2 \pi} \int_{-\sigma}^{\sigma}(i \tau)^{k}\left(1+\frac{n}{n-k}\left(\frac{n}{k}\right)^{\frac{k}{n-k}}\left(\frac{\tau}{\sigma_{0}}\right)^{2 n}\right)^{-1} y(\tau) e^{i \tau t} d \tau,
$$

где $\sigma_{0}=\min (\sigma, \hat{\sigma})$, - оптимальный метод.

Если $k=0$ u $0<\sigma<\infty$, то

$$
E\left(x(\cdot), C_{2}^{n}, I_{2}^{\delta, \sigma}\right)=\sqrt{\frac{\delta^{2}}{2 \pi}+\frac{1}{\sigma^{2 n}}}
$$

$u$

$$
\widehat{\varphi}(y)(t)=\frac{1}{2 \pi} \int_{-\sigma}^{\sigma}\left(1+\left(\frac{\tau}{\sigma}\right)^{2 n}\right)^{-1} y(\tau) e^{i \tau t} d \tau
$$

- оптимальный метод.

Из теоремы 2 следует, что в задаче (2) при $p=2$ также наблюдается эффект «насыщения». Здесь аналогом соотношения (8) является неравенство

$$
\delta^{2} \sigma^{2 n} \leqslant 2 \pi\left(\frac{n}{k}\right)^{\frac{n}{n-k}}
$$

при нарушении которого имеющаяся информация оказывается избыточной.

Отметим, что метод

$$
\widetilde{\varphi}(y)(t)=\frac{1}{2 \pi} \int_{-\sigma}^{\sigma}(i \tau)^{k} y(\tau) e^{i \tau t} d \tau,
$$

сопоставляющий функции $y(\cdot) k$-ю производную ее обратного преобразования Фурье, при $k=0$ также оптимален (наряду с методом (9)) в задаче восстановления из теоремы 2. Это легко установить непосредственной оценкой. Однако можно показать, что при $k>0$ этот, на первый взгляд, естественный метод уже не оптимален и, более того, его погрешность равна $\infty$.

Tеорема 3. Пусть $n \in \mathbb{N}, 0 \leqslant k \leqslant n-1 u 2 \leqslant p \leqslant \infty$. Имеет место точное неравенство

$$
\left\|x^{(k)}(\cdot)\right\|_{L_{2}(\mathbb{R})} \leqslant K(k, n, p)\|F x(\cdot)\|_{L_{p}(\mathbb{R})}^{\frac{n-k}{n+1 / 2-1 / p}}\left\|x^{(n)}(\cdot)\right\|_{L_{2}(\mathbb{R})}^{\frac{k+1 / 2-1 / p}{n+1 / 2-1 / p}},
$$

где при $2<p<\infty$

$$
\begin{gathered}
K(k, n, p)=\sqrt{\frac{n+1 / 2-1 / p}{k+1 / 2-1 / p}}\left(\frac{\sqrt{k+1 / 2-1 / p} B^{1 / 2-1 / p}}{(2 \pi)^{1 / p}(n-k)^{1-1 / p}}\right)^{\frac{n-k}{n+1 / 2-1 / p}}, \\
B=B\left(\frac{k+1 / 2-1 / p}{(n-k)(1-2 / p)}, 2 \frac{1-1 / p}{1-2 / p}\right)
\end{gathered}
$$


и $B(\cdot, \cdot)$ есть В-функция Эйлера, $а$

$$
K(k, n, \infty)=\sqrt{\frac{2 n+1}{2 k+1}}\left(\frac{1}{\pi(2 n+1)}\right)^{\frac{n-k}{2 n+1}}, \quad K(k, n, 2)=\left(\frac{1}{2 \pi}\right)^{\frac{n-k}{2 n}} .
$$

При $p=2$ неравенство (10) совпадает (в силу теоремы Планшереля) с известным неравенством Харди-Литтлвуда-Полиа. Неравенства вида (10) с разными метриками, где вместо преобразования Фурье функции стоит сама функция, обычно называют неравенствами для производных колмогоровского типа. Они играют важную роль в различных вопросах анализа и теории приближений. Им посвящена большая литература (см., например, $[11,12])$.

\section{§3. Доказательства}

Начнем с одного простого утверждения, касающегося оценки снизу погрешности оптимального восстановления.

Лемма 1. Пусть в задаче (1) множество

$$
\operatorname{gr} I=\{(x, y) \in X \times Y: x \in C, y \in I(x)\}
$$

центтрально-симметрично, а множество

$$
I^{-1}(0)=\{x \in C: 0 \in I(x)\}
$$

непусто. Тогда

$$
E(\Lambda, C, I) \geqslant \sup _{x \in C, x \in I^{-1}(0)}\|\Lambda x\|_{Z} .
$$

ДокАЗАТЕЛЬСтво. Для любого метода $\varphi$ при всех $x \in C$, таких, что $x \in$ $I^{-1}(0)$, имеем

$$
2\|\Lambda x\|_{Z} \leqslant\|\Lambda x-\varphi(0)\|_{Z}+\|\Lambda(-x)-\varphi(0)\|_{Z} \leqslant 2 e(\Lambda, C, I, \varphi) .
$$

Следовательно, для любого метода $\varphi$

$$
e(\Lambda, C, I, \varphi) \geqslant \sup _{x \in C, x \in I^{-1}(0)}\|\Lambda x\|_{Z},
$$

откуда сразу же вытекает доказываемая оценка.

Доказательства теорем 1 и 2 проводятся по единой схеме. По этой причине мы сначала доказываем один общий результат (содержащий в себе соображения, связанные с подходом, основанным на принципах выпуклой оптимизации), а затем, опираясь на него, получаем упомянутые теоремы. Задача восстановления, относительно которой формулируется данный результат, является некоторой детализацией общей постановки, приведенной в начале статьи.

Пусть $T-$ конечное множество с дискретной мерой или интервал прямой (конечный или бесконечный) с мерой Лебега, $X, Y_{t}, t \in T,-$ векторные пространства с полускалярными произведениями $(\cdot, \cdot)_{X},(\cdot, \cdot)_{Y_{t}}$ и соответствующими полунормами $\|\cdot\|_{X},\|\cdot\|_{Y_{t}}, Z-$ нормированное пространство и $\delta(\cdot)$ - неотрицательная измеримая функция на $T$. Предположим, что $Y$ - некоторое подпространство функций $y(\cdot)$ на $T$ со значениями в $\bigcup_{t \in T} Y_{t}$, таких, что $y(t) \in Y_{t}$ и $t \rightarrow\|y(t)\|_{Y_{t}}-$ измеримая функция на $T$. Рассматривается задача оптимального восстановления оператора $\Lambda: X \rightarrow Z$ на классе $B X=\left\{x \in X:\|x\|_{X} \leqslant 1\right\}$ по информации о линейном операторе $I: X \rightarrow Y$, 
заданном с погрешностью $\delta(\cdot)$. Точнее говоря, для каждого $x \in B X$ известна функция $y(\cdot) \in Y$, такая, что для п. в. $t \in T$

$$
\|\operatorname{Ix}(t)-y(t)\|_{Y_{t}} \leqslant \delta(t) .
$$

Погрешность оптимального восстановления записывается в этом случае в виде

$$
E(\Lambda, B X, I, \delta(\cdot))=\inf _{\varphi: Y \rightarrow Z} \sup _{\substack{x \in B X, y(\cdot) \in Y \\\|I x(t)-y(t)\|_{Y_{t}} \leqslant \delta(t)}}\|\Lambda x-\varphi(y)\|_{Z} .
$$

Нас интересует значение этой величины и оптимальный метод.

Из леммы 1 следует, что

$$
E(\Lambda, B X, I, \delta(\cdot)) \geqslant \sup _{\substack{x \in B X \\\|I x(t)\|_{Y_{t} \leqslant \delta(t)} \text { п. в. }}}\|\Lambda x\|_{Z} \cdot
$$

Рассмотрим следующую экстремальную задачу (значение которой совпадает с квадратом величины, стоящей в правой части неравенства (14)):

$$
\|\Lambda x\|_{Z}^{2} \rightarrow \max , \quad\|I x(t)\|_{Y_{t}}^{2} \leqslant \delta^{2}(t) \text { для п. в. } t \in T, \quad\|x\|_{X}^{2} \leqslant 1 .
$$

Положим

$$
\mathscr{L}\left(x, \lambda_{1}(\cdot), \lambda_{2}\right)=-\|\Lambda x\|_{Z}^{2}+\int_{T} \lambda_{1}(t)\|I x(t)\|_{Y_{t}}^{2} d \mu+\lambda_{2}\|x\|_{X}^{2},
$$

где $\lambda_{1}(\cdot)-$ измеримая неотрицательная функция, $\lambda_{2} \geqslant 0$, а $d \mu-$ мера Лебега, если $T$ - интервал прямой, и дискретная мера, если $T-$ конечное множество.

Теорема 4. Предположим, ито существуют измеримая неотрицательная функция $\hat{\lambda}_{1}(\cdot)$ и число $\widehat{\lambda}_{2} \geqslant 0$, такие, что функция $\hat{\lambda}_{1}(\cdot) \delta^{2}(\cdot)$ и функции $t \rightarrow \widehat{\lambda}_{1}(t)\left(y^{1}(t), y^{2}(t)\right)_{Y_{t}}$ при всех $y^{1}(\cdot), y^{2}(\cdot) \in Y$ суммируемы на $T u$

(a) $\mathscr{L}\left(x, \widehat{\lambda}_{1}(\cdot), \widehat{\lambda}_{2}\right) \geqslant 0 \quad \forall x \in X$.

Пусть, кроме того, существует такая последовательность $\left\{x_{m}\right\}$ допустимых элементов в (15), ито выполнены условия

(b) $\lim _{m \rightarrow \infty} \mathscr{L}\left(x_{m}, \widehat{\lambda}_{1}(\cdot), \widehat{\lambda}_{2}\right)=0$,

(c) $\lim _{m \rightarrow \infty}\left(\int_{T} \widehat{\lambda}_{1}(t)\left(\left\|I x_{m}(t)\right\|_{Y_{t}}^{2}-\delta^{2}(t)\right) d \mu+\widehat{\lambda}_{2}\left(\left\|x_{m}\right\|_{X}^{2}-1\right)\right)=0$.

Тогда если $x_{y}$ - решение экстремальной задачи

$$
\int_{T} \widehat{\lambda}_{1}(t)\|I x(t)-y(t)\|_{Y_{t}}^{2} d \mu+\widehat{\lambda}_{2}\|x\|_{X}^{2} \rightarrow \min , \quad x \in X,
$$

mo

$$
\varphi(y)=\Lambda x_{y}
$$

- оптимальный метод восстановления и

$$
E(\Lambda, B X, I, \delta(\cdot))=\sup _{\substack{x \in B X \\\|I x(t)\|_{Y_{t}} \leqslant \delta(t) \text { п. в. }}}\|\Lambda x\|_{Z}=\sqrt{\int_{T} \widehat{\lambda}_{1}(t) \delta^{2}(t) d \mu+\widehat{\lambda}_{2}} .
$$


ДокАЗАТЕЛЬСтво. 1. Покажем, что значения задачи (15) и задачи

$$
\|\Lambda x\|_{Z}^{2} \rightarrow \max , \quad \int_{T} \widehat{\lambda}_{1}(t)\|I x(t)\|_{Y_{t}}^{2} d \mu+\widehat{\lambda}_{2}\|x\|_{X}^{2} \leqslant S
$$

где

$$
S=\int_{T} \widehat{\lambda}_{1}(t) \delta^{2}(t) d \mu+\widehat{\lambda}_{2},
$$

совпадают и равны $S$. Действительно, для любого допустимого в (15) элемента $x \in X$ имеем с учетом (а)

$$
-\|\Lambda x\|_{Z}^{2} \geqslant-\|\Lambda x\|_{Z}^{2}+\int_{T} \widehat{\lambda}_{1}(t)\left(\|I x(t)\|_{Y_{t}}^{2}-\delta^{2}(t)\right) d \mu+\widehat{\lambda}_{2}\left(\|x\|_{X}^{2}-1\right) \geqslant-S .
$$

С другой стороны, используя последовательно (c) и (b), получаем, что

$$
\begin{aligned}
-\lim _{m \rightarrow \infty}\left\|\Lambda x_{m}\right\|^{2}= & \lim _{m \rightarrow \infty}\left(-\left\|\Lambda x_{m}\right\|_{Z}^{2}\right. \\
& \left.+\int_{T} \widehat{\lambda}_{1}(t)\left(\left\|I x_{m}(t)\right\|_{Y_{t}}^{2}-\delta^{2}(t)\right) d \mu+\widehat{\lambda}_{2}\left(\left\|x_{m}\right\|_{X}^{2}-1\right)\right)=-S,
\end{aligned}
$$

т. е. $S-$ значение задачи (15). Но, очевидно, эти же рассуждения доказывают, что $S$ является и значением задачи (19).

2. Оценка сверху. Рассмотрим векторное пространство $H=X \times Y$ с полускалярным произведением

$$
\left(\left(x^{1}, y^{1}(\cdot)\right),\left(x^{2}, y^{2}(\cdot)\right)\right)_{H}=\int_{T} \widehat{\lambda}_{1}(t)\left(y^{1}(t), y^{2}(t)\right)_{Y_{t}} d \mu+\widehat{\lambda}_{2}\left(x^{1}, x^{2}\right)_{X} .
$$

Тогда экстремальная задача (16) может быть переписана в виде

$$
\|(x, \operatorname{Ix}(\cdot))-(0, y(\cdot))\|_{H}^{2} \rightarrow \min , \quad x \in X .
$$

Если $x_{y}-$ решение этой задачи, то легко показать, что для всех $x \in X$ выполняется равенство

$$
\left(\left(x_{y}, I x_{y}(\cdot)\right)-(0, y(\cdot)),(x, I x(\cdot))\right)_{H}=0 .
$$

Отсюда следует, что

$\|(x, I x(\cdot))-(0, y(\cdot))\|_{H}^{2}=\left\|(x, I x(\cdot))-\left(x_{y}, I x_{y}(\cdot)\right)\right\|_{H}^{2}+\left\|\left(x_{y}, I x_{y}(\cdot)\right)-(0, y(\cdot))\right\|_{H}^{2}$. Если $\|x\|_{X} \leqslant 1$ и $\|I x(t)-y(t)\|_{Y_{t}} \leqslant \delta(t), t \in T$, то из последнего неравенства вытекает, что

$$
\begin{aligned}
\left\|(x, I x(\cdot))-\left(x_{y}, I x_{y}(\cdot)\right)\right\|_{H}^{2} & \leqslant\|(x, I x(\cdot))-(0, y(\cdot))\|_{H}^{2} \\
& =\int_{T} \widehat{\lambda}_{1}(t)\|I x(t)-y(t)\|_{Y_{t}}^{2} d \mu+\widehat{\lambda}_{2}\|x\|_{X}^{2} \leqslant S .
\end{aligned}
$$

Полагая $z=x-x_{y}$, приходим к неравенству

и, значит,

$$
\int_{T} \widehat{\lambda}_{1}(t)\|I z(t)\|_{Y_{t}}^{2} d \mu+\widehat{\lambda}_{2}\|z\|_{X}^{2} \leqslant S
$$

$$
\left\|\Lambda x-\Lambda x_{y}\right\|_{Z}=\|\Lambda z\|_{Z} \leqslant \sup _{\int_{T} \widehat{\lambda}_{1}(t)\left\|I x_{t}\right\|_{Y_{t}}^{2} d \mu+\widehat{\lambda}_{2}\|x\|_{X}^{2} \leqslant S}\|\Lambda x\|_{Z}=\sqrt{S} .
$$


Учитывая (14), получаем равенство (18) и оптимальность метода (17).

ДокАЗАТЕЛЬСтво теОРемы 1 . Пусть сначала $\sigma_{0}<\infty$. ЗаПишем рассматриваемую задачу в терминах теоремы 4. Здесь $T=\Delta_{\sigma}, X=X_{\infty}^{n}-$ векторное пространство с полускалярным произведением

$$
\left(x_{1}(\cdot), x_{2}(\cdot)\right)_{X_{\infty}^{n}}=\int_{\mathbb{R}} x_{1}^{(n)}(t) \overline{x_{2}^{(n)}(t)} d t,
$$

$Y_{t}=\mathbb{C}$ при всех $t \in \Delta_{\sigma}, Z=L_{2}(\mathbb{R}), Y=L_{\infty}\left(\Delta_{\sigma}\right), \Lambda x(\cdot)=x^{(k)}(\cdot), B X=C_{\infty}^{n}$ и оператор $I: X_{\infty}^{n} \rightarrow L_{\infty}\left(\Delta_{\sigma}\right)$ определен равенством $\operatorname{Ix}(\cdot)=F x(\cdot)$. При таких обозначениях задача $(3)$ совпадает с задачей $(13)$. Функция $\mathscr{L}\left(x(\cdot), \lambda_{1}(\cdot), \lambda_{2}\right)$ из теоремы 4 имеет в этом случае вид

$$
\mathscr{L}\left(x(\cdot), \lambda_{1}(\cdot), \lambda_{2}\right)=-\left\|x^{(k)}(\cdot)\right\|_{L_{2}(\mathbb{R})}^{2}+\int_{\Delta_{\sigma}} \lambda_{1}(t)|F x(t)|^{2} d t+\lambda_{2}\left\|x^{(n)}(\cdot)\right\|_{L_{2}(\mathbb{R})}^{2} .
$$

Переходя к образам Фурье и полагая $(2 \pi)^{-1}|F x(\cdot)|^{2}=u(\cdot)$, получим (в силу теоремы Планшереля)

$$
\mathscr{L}\left(x(\cdot), \lambda_{1}(\cdot), \lambda_{2}\right)=\int_{\mathbb{R}}\left(-t^{2 k}+\lambda_{2} t^{2 n}\right) u(t) d t+2 \pi \int_{\Delta_{\sigma}} \lambda_{1}(t) u(t) d t .
$$

Положим $\widehat{\lambda}_{2}=\sigma_{0}^{-2(n-k)}$ и

$$
\widehat{\lambda}_{1}(t)= \begin{cases}(2 \pi)^{-1}\left(t^{2 k}-\widehat{\lambda}_{2} t^{2 n}\right), & |t|<\sigma_{0}, \\ 0, & |t| \geqslant \sigma_{0} .\end{cases}
$$

Тогда при всех $x(\cdot) \in X_{\infty}^{n}$

$$
\mathscr{L}\left(x(\cdot), \widehat{\lambda}_{1}(\cdot), \widehat{\lambda}_{2}\right)=\int_{|t| \geqslant \sigma_{0}}\left(-t^{2 k}+\sigma_{0} t^{2 n}\right) u(t) d t \geqslant 0 .
$$

Положим

$$
\gamma=1-\frac{1}{2 \pi} \int_{-\sigma_{0}}^{\sigma_{0}} t^{2 n} \delta^{2}(t) d t
$$

Если $\gamma=0$, то, обозначив через $\hat{x}(\cdot)$ обратное преобразование Фурье функции, совпадающей с $\delta(\cdot)$ в интервале $\left(-\sigma_{0}, \sigma_{0}\right)$ и равной нулю вне него, легко проверить, что условия (b) и (c) теоремы 4 выполнены для постоянной последовательности $x_{m}(\cdot)=\hat{x}(\cdot)$.

Если $\gamma>0$ (в этом случае, очевидно, $\sigma_{0}=\sigma$ ), то положим для $m>\gamma^{-2}$

$$
u_{m}(t)= \begin{cases}\delta^{2}(t) /(2 \pi), & |t|<\sigma, \\ \sigma^{-2 n}(m \gamma-\sqrt{m}) / 2, & \sigma \leqslant|t| \leqslant \sigma+1 / m, \\ 0, & |t|>\sigma+1 / m\end{cases}
$$

(через $x_{m}(\cdot)$ обозначим обратное преобразование Фурье функции $\sqrt{2 \pi u_{m}(\cdot)}$ ). Нетрудно убедиться, что

$$
\lim _{m \rightarrow \infty} \mathscr{L}\left(x_{m}(\cdot), \widehat{\lambda}_{1}(\cdot), \widehat{\lambda}_{2}\right)=0 .
$$

Кроме того,

$$
\int_{-\sigma}^{\sigma} \widehat{\lambda}_{1}(t)\left(2 \pi u_{m}(t)-\delta^{2}(t)\right) d t=0
$$


И

$$
\begin{aligned}
\left\|x_{m}^{(n)}(\cdot)\right\|_{L_{2}(\mathbb{R})}^{2} & =\int_{\mathbb{R}} t^{2 n} u_{m}(t) d t=\frac{1}{2 \pi} \int_{-\sigma}^{\sigma} t^{2 n} \delta^{2}(t) d t+\frac{m \gamma-\sqrt{m}}{\sigma^{2 n}} \int_{\sigma}^{\sigma+1 / m} t^{2 n} d t \\
& =1-\gamma+\frac{(m \gamma-\sqrt{m})\left((\sigma+1 / m)^{2 n+1}-\sigma^{2 n+1}\right)}{\sigma^{2 n}(2 n+1)} \stackrel{m \rightarrow \infty}{\longrightarrow} 1 .
\end{aligned}
$$

Из последних равенств следует также, что при достаточно больших $m$

$$
\left\|x_{m}^{(n)}(\cdot)\right\|_{L_{2}(\mathbb{R})}^{2}<1-\frac{1}{2 \sqrt{m}}
$$

т. е. функции $x_{m}(\cdot)$ являются допустимыми в задаче $(15)$.

Задача $(16)$ для $y(\cdot) \in L_{\infty}\left(\Delta_{\sigma}\right)$ имеет вид

$$
\int_{\Delta_{\sigma}} \widehat{\lambda}_{1}(t)|F x(t)-y(t)|^{2} d t+\widehat{\lambda}_{2}\left\|x^{(n)}(\cdot)\right\|_{L_{2}(\mathbb{R})} \rightarrow \min , \quad x(\cdot) \in X_{\infty}^{n}
$$

С учетом теоремы Планшереля она может быть записана так:

$$
\int_{\Delta_{\sigma}} \widehat{\lambda}_{1}(t)|F x(t)-y(t)|^{2} d t+\frac{\widehat{\lambda}_{2}}{2 \pi} \int_{\mathbb{R}} t^{2 n}|F x(t)|^{2} d t \rightarrow \min , \quad x(\cdot) \in X_{\infty}^{n} .
$$

Нетрудно проверить, что ее решением является функция $\hat{x}_{y}(\cdot)$, такая, что

$$
F \hat{x}_{y}(t)= \begin{cases}\frac{2 \pi \widehat{\lambda}_{1}(t)}{2 \pi \widehat{\lambda}_{1}(t)+\widehat{\lambda}_{2} t^{2 n}} y(t), & |t|<\sigma_{0} \\ 0, & |t| \geqslant \sigma_{0}\end{cases}
$$

T. e.

$$
F \hat{x}_{y}(t)= \begin{cases}\left(1-\sigma_{0}^{-2(n-k)} t^{2(n-k)}\right) y(t), & |t|<\sigma_{0} \\ 0, & |t| \geqslant \sigma_{0}\end{cases}
$$

Из теоремы 4 вытекает, что оптимальный метод имеет вид (5), а для его погрешности справедлива формула (4).

Если $\sigma_{0}=\infty$ (при этом, очевидно, $\sigma=\infty$ ), то из леммы 1 , обозначив через $\hat{x}(\cdot)$ обратное преобразование Фурье функции $\delta(\cdot)$, получим

$$
\begin{aligned}
E\left(x^{(k)}(\cdot), C_{\infty}^{n}, I^{\delta(\cdot), \infty}\right) & \geqslant \sup _{\substack{x(\cdot) \in C_{\infty}^{n} \\
|F x(t)| \leqslant \delta(t) \text { п. в. }}}\left\|x^{(k)}(\cdot)\right\|_{L_{2}(\mathbb{R})} \\
& \geqslant\left\|\hat{x}^{(k)}(\cdot)\right\|_{L_{2}(\mathbb{R})}=\sqrt{\frac{1}{2 \pi} \int_{-\infty}^{\infty} t^{2 k} \delta^{2}(t) d t .}
\end{aligned}
$$


С другой стороны, для метода (7) имеем

$$
\begin{aligned}
& e\left(x^{(k)}(\cdot), C_{\infty}^{n}, I^{\delta(\cdot), \infty}, \widehat{\varphi}\right) \sup _{\substack{x(\cdot) \in C_{\infty}^{n}, y(\cdot) \in L_{\infty}(\mathbb{R}) \\
|F x(t)-y(t)| \leqslant \delta(t) \text { п. в. }}}\left\|x^{(k)}(\cdot)-\widehat{\varphi}(y)(\cdot)\right\|_{L_{2}(\mathbb{R})} \\
&= \sup _{\substack{x(\cdot) \in C_{\infty}^{n}, y(\cdot) \in L_{\infty}(\mathbb{R}) \\
|F x(t)-y(t)| \leqslant \delta(t) \text { п. в. }}}\left(\frac{1}{2 \pi} \int_{-\infty}^{\infty} t^{2 k}|F x(t)-y(t)|^{2} d t\right)^{1 / 2} \\
& \leqslant \sqrt{\frac{1}{2 \pi} \int_{-\infty}^{\infty} t^{2 k} \delta^{2}(t) d t .}
\end{aligned}
$$

Из теоремы 1 , в частности, вытекает, что если $\delta(t) \equiv \delta>0$ и $\sigma=\infty$ (см. следствие 1), то

$$
\sup _{\substack{x(\cdot) \in C_{\infty}^{n} \\|F x(t)| \leqslant \delta(t) \text { п. в. }}}\left\|x^{(k)}(\cdot)\right\|_{L_{2}(\mathbb{R})}=K(k, n, \infty) \delta^{\frac{2(n-k)}{2 n+1}},
$$

где константа $K(k, n, \infty)$ определена равенствами $(12)$. Отсюда следует точное неравенство

$$
\left\|x^{(k)}(\cdot)\right\|_{L_{2}(\mathbb{R})} \leqslant K(k, n, \infty)\|F x(\cdot)\|_{L_{\infty}(\mathbb{R})}^{\frac{2(n-k)}{2 n+1}}\left\|x^{(n)}(\cdot)\right\|_{L_{2}(\mathbb{R})}^{\frac{2 k+1}{2 n+1}} .
$$

ДоКАЗАТЕЛЬСтво теоремы 2. Снова запишем рассматриваемую задачу в терминах теоремы 4. Здесь $T$ состоит из одной точки, скажем, $t=1$. Далее, $X=X_{2}^{n}$ - векторное пространство с полускалярным произведением

$$
\left(x_{1}(\cdot), x_{2}(\cdot)\right)_{X_{2}^{n}}=\int_{\mathbb{R}} x_{1}^{(n)}(t) \overline{x_{2}^{(n)}(t)} d t,
$$

$Y_{1}=Y=L_{2}\left(\Delta_{\sigma}\right), Z=L_{2}(\mathbb{R}), \Lambda x(\cdot)=x^{(k)}(\cdot), B X=C_{2}^{n}$, информационный оператор $I: X_{2}^{n} \rightarrow L_{2}\left(\Delta_{\sigma}\right)$ определен равенством $I x(\cdot)=F x(\cdot)$ и $\delta(1)=\delta>0$. Функция $\mathscr{L}\left(x(\cdot), \lambda_{1}, \lambda_{2}\right)$ из теоремы 4 имеет здесь вид

$$
\mathscr{L}\left(x(\cdot), \lambda_{1}, \lambda_{2}\right)=-\left\|x^{(k)}(\cdot)\right\|_{L_{2}(\mathbb{R})}^{2}+\lambda_{1} \int_{\Delta_{\sigma}}|F x(t)|^{2} d t+\lambda_{2}\left\|x^{(n)}(\cdot)\right\|_{L_{2}(\mathbb{R})}^{2} .
$$

Переходя к образам Фурье и полагая $(2 \pi)^{-1}|F x(\cdot)|^{2}=u(\cdot)$, получим

$$
\mathscr{L}\left(x(\cdot), \lambda_{1}, \lambda_{2}\right)=\int_{\mathbb{R}}\left(-t^{2 k}+2 \pi \lambda_{1} \chi_{\sigma}(t)+\lambda_{2} t^{2 n}\right) u(t) d t,
$$

где $\chi_{\sigma}(\cdot)-$ характеристическая функция интервала $\Delta_{\sigma}$.

Положим

$$
\begin{gathered}
\widehat{\lambda}_{1}= \begin{cases}\frac{1}{2 \pi}\left(\frac{k}{n}\right)^{\frac{k}{n-k}} \frac{n-k}{n} \sigma_{0}^{2 k}, & k>0, \\
\frac{1}{2 \pi}, & \widehat{\lambda}_{2}= \begin{cases}\sigma_{0}^{-2(n-k)}, & k>0, \\
\sigma^{-2 n}, & k=0,\end{cases} \end{cases} \\
\hat{t}= \begin{cases}\left(\frac{k}{n}\right)^{\frac{1}{2(n-k)} \sigma_{0},} & k>0, \\
0, & k=0 .\end{cases}
\end{gathered}
$$


Нетрудно проверить, что при всех $x(\cdot) \in X_{2}^{n}$

$$
\mathscr{L}\left(x(\cdot), \widehat{\lambda}_{1}, \widehat{\lambda}_{2}\right) \geqslant 0 .
$$

Предположим сначала, что $k>0$ и $\sigma<\hat{\sigma}$ (в этом случае $\sigma_{0}=\sigma$ ). Рассмотрим для достаточно больших $m$ последовательность функций

$$
u_{m}(t)= \begin{cases}m \frac{\delta^{2}}{4 \pi}, & \hat{t} \leqslant|t| \leqslant \hat{t}+\frac{1}{m}, \\ \frac{m-\sqrt{m}}{2 \sigma^{2 n}}\left(1-\frac{\delta^{2}}{2 \pi} \hat{t}^{2 n}\right), & \sigma \leqslant|t| \leqslant \sigma+\frac{1}{m}, \\ 0 & \text { в остальных случаях. }\end{cases}
$$

Тогда

$$
\begin{aligned}
2 \pi \int_{\Delta_{\sigma}} u_{m}(t) d t & =\delta^{2} \\
\int_{\mathbb{R}} t^{2 n} u_{m}(t) d t= & \frac{m \delta^{2}}{2 \pi} \frac{(\hat{t}+1 / m)^{2 n+1}-\hat{t}^{2 n+1}}{2 n+1} \\
& \quad+\frac{m-\sqrt{m}}{2 \sigma^{2 n}}\left(1-\frac{\delta^{2}}{2 \pi} \hat{t}^{2 n}\right) \frac{(\sigma+1 / m)^{2 n+1}-\sigma^{2 n+1}}{2 n+1} \stackrel{m \rightarrow \infty}{\longrightarrow} 1 .
\end{aligned}
$$

Кроме того, при достаточно больших $m$

$$
\int_{\mathbb{R}} t^{2 n} u_{m}(t) d t<1-\left(1-\frac{\delta^{2}}{2 \pi} \hat{t}^{2 n}\right) \frac{1}{2 \sqrt{m}} .
$$

Отсюда следует, что для функций $x_{m}(\cdot)$, являющихся обратными преобразованиями Фурье функций $\sqrt{2 \pi u_{m}(\cdot)}$, условие (c) теоремы 4 выполнено. Непосредственной проверкой можно убедиться, что условие (b) той же теоремы также выполнено.

Если $k>0$ и $\sigma \geqslant \hat{\sigma}$ или $k=0$, то следует рассмотреть функции

$$
u_{m}(t)= \begin{cases}(m-\sqrt{m}) \delta^{2} /(4 \pi), & \hat{t} \leqslant|t| \leqslant \hat{t}+1 / m, \\ 0 & \text { в остальных случаях. }\end{cases}
$$

Задача $(16)$ для $y(\cdot) \in L_{2}(\mathbb{R})$ имеет вид

$$
\widehat{\lambda}_{1}\|F x(\cdot)-y(\cdot)\|_{L_{2}\left(\Delta_{\sigma}\right)}^{2}+\widehat{\lambda}_{2}\left\|x^{(n)}(\cdot)\right\|_{L_{2}(\mathbb{R})} \rightarrow \min , \quad x(\cdot) \in X_{2}^{n} .
$$

С учетом теоремы Планшереля она может быть записана в виде

$$
\widehat{\lambda}_{1} \int_{\Delta_{\sigma}}|F x(t)-y(t)|^{2} d t+\frac{\widehat{\lambda}_{2}}{2 \pi} \int_{\mathbb{R}} t^{2 n}|F x(t)|^{2} d t \rightarrow \min , \quad x(\cdot) \in X_{2}^{n} .
$$

Решением этой задачи является функция $\hat{x}_{y}(\cdot)$, такая, что при $k>0$

$$
F \hat{x}_{y}(t)= \begin{cases}\left(1+\frac{n}{n-k}\left(\frac{n}{k}\right)^{\frac{k}{n-k}}\left(\frac{t}{\sigma_{0}}\right)^{2 n}\right)^{-1} y(t), & |t|<\sigma, \\ 0, & |t| \geqslant \sigma,\end{cases}
$$


а при $k=0$

$$
F \hat{x}_{y}(t)= \begin{cases}\left(1+\left(\frac{t}{\sigma}\right)^{2 n}\right)^{-1} y(t), & |t|<\sigma \\ 0, & |t| \geqslant \sigma .\end{cases}
$$

Теперь доказываемая теорема вытекает из теоремы 4.

Из теоремы 2 вытекает, что

$$
\sup _{\substack{x(\cdot) \in C_{2}^{n} \\\|F x(\cdot)\|_{L_{2}(\mathbb{R})} \leqslant \delta}}\left\|x^{(k)}(\cdot)\right\|_{L_{2}(\mathbb{R})}=\left(\frac{\delta^{2}}{2 \pi}\right)^{\frac{n-k}{2 n}},
$$

откуда следует точное неравенство

$$
\left\|x^{(k)}(\cdot)\right\|_{L_{2}(\mathbb{R})} \leqslant\left(\frac{1}{2 \pi}\right)^{\frac{n-k}{2 n}}\|F x(\cdot)\|_{L_{2}(\mathbb{R})}^{\frac{n-k}{n}}\left\|x^{(n)}(\cdot)\right\|_{L_{2}(\mathbb{R})}^{\frac{k}{n}} .
$$

ДОКАЗАТЕЛЬСТво ТЕОРЕМЫ 3. Случаи $p=\infty, 2$ вытекают из теорем 1,2 . Будем считать, что $2<p<\infty$. Рассмотрим экстремальную задачу

$$
\left\|x^{(k)}(\cdot)\right\|_{L_{2}(\mathbb{R})}^{2} \rightarrow \max , \quad\|F x(\cdot)\|_{L_{p}(\mathbb{R})}^{2} \leqslant \delta^{2}, \quad\left\|x^{(n)}(\cdot)\right\|_{L_{2}(\mathbb{R})}^{2} \leqslant 1 .
$$

В образах Фурье, положив $u(\cdot)=(2 \pi)^{-1}|F x(\cdot)|^{2}$, эту задачу можно переписать в виде

$$
\int_{\mathbb{R}} t^{2 k} u(t) d t \rightarrow \max , \quad \int_{\mathbb{R}} u^{p / 2}(t) d t \leqslant \frac{\delta^{p}}{2 \pi}, \quad \int_{\mathbb{R}} t^{2 n} u(t) d t \leqslant 1, u(t) \geqslant 0 .
$$

Свяжем с задачей (20) следующую функцию Лагранжа:

$$
\mathscr{L}\left(u(\cdot), \lambda_{1}, \lambda_{2}\right)=\int_{\mathbb{R}}\left(-t^{2 k} u(t)+\lambda_{1} u^{p / 2}(t)+\lambda_{2} t^{2 n} u(t)\right) d t .
$$

Если мы найдем такую допустимую в $(20)$ функцию $\widehat{u}(\cdot)$ и такие множители Лагранжа $\widehat{\lambda}_{1}, \widehat{\lambda}_{2} \geqslant 0$, что

(a) $\min _{u(t) \geqslant 0} \mathscr{L}\left(u(\cdot), \widehat{\lambda}_{1}, \widehat{\lambda}_{2}\right)=\mathscr{L}\left(\widehat{u}(\cdot), \widehat{\lambda}_{1}, \widehat{\lambda}_{2}\right)$,

(b) $\widehat{\lambda}_{1}\left(\int_{\mathbb{R}} u(t)^{p / 2} d t-\frac{\delta^{p}}{2 \pi}\right)=0$,

(c) $\widehat{\lambda}_{2}\left(\int_{\mathbb{R}} t^{2 n} u(t) d t-1\right)=0$,

то $\widehat{u}(\cdot)-$ решение задачи $(20)$. Действительно, для любой допустимой функции $u(\cdot)$ имеем

$$
\begin{aligned}
-\int_{\mathbb{R}} t^{2 k} u(t) d t & \geqslant-\int_{\mathbb{R}} t^{2 k} u(t) d t+\widehat{\lambda}_{1}\left(\int_{\mathbb{R}} u(t)^{p / 2} d t-\frac{\delta^{p}}{2 \pi}\right)+\widehat{\lambda}_{2}\left(\int_{\mathbb{R}} t^{2 n} u(t) d t-1\right) \\
& \geqslant-\int_{\mathbb{R}} t^{2 k} \widehat{u}(t) d t+\widehat{\lambda}_{1}\left(\int_{\mathbb{R}} \widehat{u}(t)^{p / 2} d t-\frac{\delta^{p}}{2 \pi}\right)+\widehat{\lambda}_{2}\left(\int_{\mathbb{R}} t^{2 n} \widehat{u}(t) d t-1\right) \\
& =-\int_{\mathbb{R}} t^{2 k} \widehat{u}(t) d t .
\end{aligned}
$$


Положим $\hat{\lambda}_{2}=\sigma^{-2(n-k)}$, где параметр $\sigma>0$ определим позже. Тогда для всех $u(t) \geqslant 0$ и любого $\widehat{\lambda}_{1}>0$

$$
-t^{2 k} u(t)+\widehat{\lambda}_{1} u^{p / 2}(t)+\frac{t^{2 n}}{\sigma^{2(n-k)}} u(t) \geqslant-t^{2 k} \widehat{u}(t)+\widehat{\lambda}_{1} \widehat{u}^{p / 2}(t)+\frac{t^{2 n}}{\sigma^{2(n-k)}} \widehat{u}(t),
$$

где

$$
\widehat{u}(t)= \begin{cases}\left(\frac{2}{p \widehat{\lambda}_{1}}\left(t^{2 k}-\frac{t^{2 n}}{\sigma^{2(n-k)}}\right)\right)^{\frac{1}{p / 2-1}}, & |t| \leqslant \sigma, \\ 0, & |t|>\sigma .\end{cases}
$$

Тем самым выполнено условие (а). Выберем $\sigma$ и $\hat{\lambda}_{1}$ так, чтобы удовлетворить условиям (b) и (c):

$$
\begin{aligned}
& \left(\frac{2}{p \widehat{\lambda}_{1}}\right)^{\frac{p / 2}{p / 2-1}} \int_{-\sigma}^{\sigma}\left(t^{2 k}-\frac{t^{2 n}}{\sigma^{2(n-k)}}\right)^{\frac{p / 2}{p / 2-1}} d t=\frac{\delta^{p}}{2 \pi} \\
& \left(\frac{2}{p \widehat{\lambda}_{1}}\right)^{\frac{1}{p / 2-1}} \int_{-\sigma}^{\sigma} t^{2 n}\left(t^{2 k}-\frac{t^{2 n}}{\sigma^{2(n-k)}}\right)^{\frac{1}{p / 2-1}} d t=1 .
\end{aligned}
$$

Сделав замену $t=\sigma y$ и выразив полученные интегралы через значение бетафункции $B$, определенное равенством (11), получим

$$
\begin{gathered}
\sigma^{\frac{p k}{p / 2-1}+1}\left(\frac{2}{p \widehat{\lambda}_{1}}\right)^{\frac{p / 2}{p / 2-1}} \frac{B}{n-k}=\frac{\delta^{p}}{2 \pi}, \\
\sigma^{\frac{2 k}{p / 2-1}+2 n+1}\left(\frac{2}{p \widehat{\lambda}_{1}}\right)^{\frac{1}{p / 2-1}} \frac{(k+1 / 2-1 / p) B}{(n-k)^{2}}=1 .
\end{gathered}
$$

Отсюда

$$
\left(\frac{2}{p \widehat{\lambda}_{1}}\right)^{\frac{1}{p / 2-1}}=\frac{(n-k)^{2}}{(k+1 / 2-1 / p) B} \sigma^{-\frac{2 k}{p / 2-1}-2 n-1}
$$

и

$$
\sigma=\left(\frac{(2 \pi)^{1 / p}(n-k)^{1-1 / p}}{\delta(k+1 / 2-1 / p)^{1 / 2} B^{1 / 2-1 / p}}\right)^{\frac{1}{n+1 / 2-1 / p}} .
$$

Учитывая (21), будем иметь

$$
\int_{\mathbb{R}} t^{2 k} \widehat{u}(t) d t=\frac{n+1 / 2-1 / p}{k+1 / 2-1 / p} \sigma^{-2(n-k)} .
$$

Подставив значение $\sigma$ из (22), получим, что при всех $2<p<\infty$

$$
\sup _{\substack{x(\cdot) \in F_{p}^{n} \\\|F x(\cdot)\|_{L_{p}(\mathbb{R})} \leqslant \delta}}\left\|x^{(k)}(\cdot)\right\|_{L_{2}(\mathbb{R})}=K \delta^{\frac{n-k}{n+1 / 2-1 / p}} .
$$

Из этого равенства непосредственно вытекает доказываемое точное неравенство для производных. 


\section{ЛИТЕРАТУРА}

1. Смоляк $C$. $A$. Об оптимальном восстановлении функций и функционалов от них. Канд. дисс., МГУ, М., 1965.

2. Micchelli C. A., Rivlin T. J. A survey of optimal recovery. In: Optimal Estimation in Approximation Theory (C. A. Micchelli and T. J. Rivlin, eds.). Plenum Press, New York, 1977, pp. 1-54.

3. Traub J. F., Woźniakowski H. A General Theory of Optimal Algorithms. Academic Press, New York, 1980.

4. Micchelli C. A., Rivlin T. J. Lectures on Optimal Recovery. Lect. Notes in Math., Vol. 1129, Springer-Verlag, Berlin, 1985, pp. 21-93.

5. Osipenko K. Yu. Optimal Recovery of Analytic Functions. Nova Science Publ., Inc., Huntington, New York, 2000.

6. Магарил-Ильяев Г. Г., Тихомиров В. М. О неравенствах для производных колмогоровского типа. Матем. сб., 188. № 12. 73-106 (1997).

7. Магарил-Ильяев Г. Г., Тихомиров В. М. Выпуклый анализ и его приложения. Эдиториал УРСС, М., 2000.

8. Магарил-Ильяев Г. Г., Осипенко К. Ю., Тихомиров В. М. Оптимальное восстановление и теория экстремума. Докл. РАН, 379. №2. 161-164 (2001).

9. Melkman A. A., Micchelli $C$. A. Optimal estimation of linear operators in Hilbert spaces from inaccurate data. SIAM J. Numer. Anal., 16. 87-105 (1979).

10. Магарил-Ильяев Г. Г., Осипенко К. Ю. Оптимальное восстановление функций и их производных по коэффициентам Фурье, заданным с погрешностью. Матем. сб., 193, 79-100 (2002).

11. Тихомиров В. М., Магарил-Ильяев Г. Г. Неравенства для производных. В кн.: Избранные труды А. Н. Колмогорова. Математика и механика. Наука, М., 1985, с. 386390.

12. Арестов B. В. Приближение неограниченных операторов ограниченными и родственные экстремальные задачи. УМН, 51, 89-124 (1996).

Московский государственный институт радиотехники, электроники и автоматики

Поступило в редакцию

МАТИ - Российский государственный технологический

22 июля 2002 г. университет им. К. Э. Циолковского 